\title{
Answer to a Quiz on page 23: Electrophysiology
}

Correct answer 3: pre-excited atrial fibrillation

\section{Comment:}

In patients with an accessory pathway a short antegrade refractory period can result in atrial fibrillation with rapid, irregular, ventricular activation via the accessory pathway that can result in hemodynamic compromise and can trigger ventricular fibrillation. Scar-related ventricular tachycardia involves a reentrant circuit and usually presents as a regular wide complex tachycardia. Typical atrial flutter with 1:1 conduction over the accessory pathway would also present as a regular wide complex tachycardia, with an expected rate around 300/min. Antidromic AVRT would also present as a wide complex regular tachycardia as the antegrade part of the circuit is over the accessory pathway, retrograde conduction over the atrioventricular node.

Ayan Abdrakhmanov, Zhandos Esilbayev Department of Interventional Arrhythmology

National Research Cardiac Surgery Center Astana, Kazakhstan

Peer-review: Internal

Authorship: A.A. and Z.E. equally contrıbuted to preparation of quiz Conflict of interest: None to declare Acknowledgement and funding: None to declare

Address for Correspondence: Ayan Abdrakhmanov, Department of Interventional Arrhythmology, National Research Cardiac Surgery Center Astana, Kazakhstan, Email: ayan-3@mail.ru 